\title{
Macroparticle model for longitudinal emittance growth caused by negative mass instability in a proton synchrotron
}

\author{
J. A. MacLachlan \\ Fermi National Accelerator Laboratory, Box 500, Batavia, Illinois 60510, USA
}

(Received 25 August 2003; published 8 April 2004)

\begin{abstract}
Both theoretical models and beam observations of negative mass instability fall short of a full description of the dynamics and the dynamical effects. Clarification by numerical modeling is now practicable because of the recent proliferation of so-called computing farms. The results of modeling reported in this paper disagree with some predictions based on a long-standing linear perturbation calculation. Validity checks on the macroparticle model are described.
\end{abstract}

DOI: 10.1103/PhysRevSTAB.7.044401

PACS numbers: 29.27.Bd, 02.60.Cb

\section{INTRODUCTION}

Most proton synchrotrons pass through an energy at which the particle circulation frequency is practically independent of momentum differences within beam bunches. At this energy, called the transition energy $\left(E_{T}\right), \partial \omega_{\text {circ }} / \partial E$ is zero, changing from positive below $E_{T}$ to negative above. The interparticle repulsion causes charge concentrations within the distribution to disperse below transition but to concentrate above. Just above transition energy, fluctuations in density are practically fixed in the bunch, and the particles in charge surplus regions push one another to higher and lower energy without much change in relative azimuth. If these perturbations of the smooth distribution constitute a sufficient peak current, the resulting field promotes the charge concentration so that the bunch emittance grows significantly within a few beam turns. The process has been studied with a linearized Vlasov equation for a bunch with elliptical phase space distribution plus a density modulation given by the statistical fluctuation for the number of beam particles present [1]. Although this analytical model is simplified from the typical case for a bunched beam with its inevitable larger scale inhomogeneities, one may hope and has hoped that the instability threshold and the relevant Fourier components of the beam current are correctly predicted. The original Hardt paper has been studied and reviewed by Ng [2], who concludes that the analysis has assumptions in need of testing in some way. Unfortunately, detailed beam observations are difficult. There is more than one mechanism for emittance growth in crossing transition, and, although microwave components can be observed in the beam current after transition crossing [3], these observations have been made at frequencies much lower than those predicted to be dominant in negative mass instability (NMI). There is no numerical relation to connect the observed microwave amplitudes to observed emittance growth. High frequency rf harmonics have been observed before transition far above the Schottky background up to several $\mathrm{GHz}$, implying microstructure or turbulence in the bunches [4]. Such components could be the seed for NMI observed in beam studies.

Efforts to elucidate the process by macroparticle beam models have been hampered by limits on macroparticle number, effectively limiting the bandwidth to 1 or 2 orders of magnitude below the top of the predicted range of unstable Fourier components of the beam current. The first paper using a macroparticle model to show bunch disruption at about the expected current threshold showed the disrupted distribution to be modulated by the binning frequency [5]. Later attempts with a few hundred to a few thousand times the 2000 macroparticles used by Lee and Teng in the original effort also evidenced this dubious property. In a recent paper by this author [6], results which did show clustering at less than the binning frequency evidenced some disagreement with the analytic model. However, despite a macroparticle count of $1.2 \times$ $10^{7}$, the calculation was manifestly statistics limited, and only qualitative conclusions were justified. The ultimate macroparticle model would employ a test particle for each particle in the real bunch. Despite the rapid increase in computing resources available for routine research, this ideal is unlikely to be attained for some years. This paper reports results using at most $6.4 \times 10^{8}$ macroparticles to simulate a bunch in the Fermilab Main Injector with intensity ranging from $6 \times 10^{10}$ to $2 \times 10^{11}$ protons in $0.2 \mathrm{eV}$ s. Because these calculations are also statistically marginal, tests have been made to establish the validity of quantitative results.

\section{BEAM PROPERTIES AND ACCELERATOR PARAMETERS}

Most of the examples reported relate to properties of the Fermilab Main Injector (FMI) which is characterized for present purposes by the parameters in Table $\mathrm{I}$. However, for a comparison to the Hardt model I have used parameters and results for the old Fermilab Main Ring (MR) from Ref. [3], also included in Table I. As may be seen, the two rings are not so very different despite the difference in circumference. These parameters are those 
TABLE I. Ring parameters used in the models.

\begin{tabular}{lcccc}
\hline \hline \multicolumn{1}{c}{ Parameter } & Symbol & FMI & MR & Units \\
\hline Circumference & $C$ & 3319.42 & 6283.19 & $\mathrm{~m}$ \\
Transition energy $/ m_{0} c^{2}$ & $\gamma_{T}$ & 21.84 & 18.85 & \\
rf peak voltage & $V_{\mathrm{rf}}$ & 3.7 & 2.5 & $\mathrm{MV}$ \\
rf harmonic & $h$ & 588.0 & 1113.0 & \\
Beam circulation frequency & $f_{0}$ & 90.2195 & 47.6478 & $\mathrm{kHz}$ \\
Ramp rate & $\dot{\gamma}$ & 240.0 & 109.94 & $\mathrm{~s}^{-1}$ \\
Synchronous phase & $\phi_{s}$ & 42.38 & 60.00 & $\mathrm{deg}$ \\
Nonadiabatic time & $T_{\text {na }}$ & 1.76 & 3.00 & $\mathrm{~ms}$ \\
Beam pipe radius & $b$ & 2.5 & 2.8 & $\mathrm{~cm}$ \\
Beam radius & $a$ & 0.4 & 0.4 & $\mathrm{~cm}$ \\
Geometric factor & $g_{0}$ & 4.66 & 4.89 & \\
Harmonic of $f_{0}$ for $g=\frac{1}{2} g_{0}$ & $n_{1 / 2}$ & 2238395.0 & 3527642.0 & \\
Number of protons per bunch & $N$ & $0.6-2.0 \times 10^{11}$ & $0.4 \times 10^{11}$ & \\
Average bunch current & $\bar{I}_{\text {bunch }}$ & $0.51-1.7$ & 0.34 & $\mathrm{~A}$ \\
\hline \hline
\end{tabular}

used in the modeling and do not agree in every particular with design parameters.

\section{VALIDATION OF THE MACROPARTICLE MODEL}

Taking the specific instance of a $4 \times 10^{10}$ proton bunch in the MR, the macroparticle model shows a factor of 2.0 emittance growth at $0.1 \mathrm{eVs}$ but just $4.6 \%$ growth at 0.12 $\mathrm{eVs}$. This outcome is consistent with Ng's evaluation of Hardt's $c$ parameter as 1.31 in the first case and 0.84 in the second because the value $c=1$ is the threshold for NMI according to Hardt's analysis.

Hardt's paper is very terse and perhaps difficult to understand for other reasons also. One of the apparent mysteries is why the threshold seems so sensitive with respect to bunch emittance. Fortunately there is an alternative, semiphenomenological, self-bunching model to establish the threshold and understand the sensitivity intuitively. Assume that any perturbation of the particle flow caused by a full microbucket entirely contained within the bunch will be negligible; for a uniform distribution this is certainly true. Then a plausible threshold criterion is

$$
H_{\mu \text { bucket }} \geq H_{b},
$$

where $H_{\mu \text { bucket }}$ is the height of the microbucket generated by the current fluctuations and $H_{b}$ is the bunch height. At wavelengths much shorter than the bunch length, the Fourier spectrum of the beam current is white noise, that is, frequency independent amplitudes independent also of large-scale bunch shape. Calculate the energy extent (bucket height) of the stable oscillation areas separately for each current component arising from the space charge impedance

$$
\frac{Z_{\|}}{n}=-i \frac{Z_{0} g}{2 \beta \gamma^{2}}:
$$

$$
V_{n}=Z_{\|} I_{n},
$$

and consequently a microbucket height in $\mathrm{eV}$ [7]:

$$
H_{\mu B, n}=\beta \sqrt{\frac{2 e V_{n} E}{\pi n|\eta|}},
$$

where $\beta$ and $\gamma$ are relativistic kinematic parameters, $Z_{0}$ is the free-space impedance of $376.7 \Omega, I_{n}$ is the $n$th harmonic peak current, $e$ is the proton charge, $E$ is the total energy of each proton, $n$ is the harmonic number with respect to the beam circulation frequency, and $\eta$ is the phase slip factor $\gamma_{T}^{-2}-\gamma^{-2}$. Because $Z_{\|}$is proportional to $n$ and $H_{\mu B, n}$ depends on $V_{n} / n$, all modes appear to become unstable at the same beam current. The growth rate of the instability is given by the small amplitude oscillation frequency in the microbuckets [7]

$$
\lambda_{\text {rise, } n}=\sqrt{\frac{n|\eta| e V_{n}}{2 \pi \beta^{2} E}} f_{0} .
$$

Thus, higher frequency modes grow faster and should dominate the progress of the disruption.

Because the instability appears first at the peak current part of the bunch, the current to be used in calculating $I_{n}$ is $\hat{I}=\bar{I}_{\text {bunch }} / B$, where $B$ is the conventional bunching factor. In the high frequency range the current can be thought of as the sum of $\delta$ functions of moving charge so each amplitude has a frequency independent factor of 2 from the Fourier expansion of the $\delta$ functions. Plausibly, therefore,

$$
I_{n}=2 \bar{I}_{\text {bunch }} / B
$$

and with this choice the stability threshold criterion of Eq. (1) gives the same results as the perturbation calculation. The heuristic model, however, does not include any limit on the action of very high frequency components, 
although at least one such limiting factor is effective, namely, the roll-off of the geometrical factor $g$. At a harmonic $n_{1 / 2}$ (see Table I) it is one-half of its low frequency value [1]:

$$
g_{n}=\frac{g_{0}}{1+\left(n / n_{1 / 2}\right)^{2}},
$$

where

$$
\begin{gathered}
g_{0}=1+2 \ln (b / a), \\
n_{1 / 2}=\gamma R_{\mathrm{eq}}\left(\frac{1.6}{b}+\frac{0.52}{a}\right),
\end{gathered}
$$

and $n$ is the harmonic number with respect to the beam circulation frequency, $R_{\text {eq }}$ is the mean orbit radius, and $a$ and $b$ are beam pipe and mean beam radii, respectively. Thus, the upper end of the active band of harmonics is limited at some point by the decrease in $g$. One may understand the sharpness of the emittance threshold in terms of whether the microbuckets are higher than the bunch or not and the insensitivity to bandwidth limitation in terms of the simultaneous instability of a wide range of modes. It has been observed in this work that the final bunch disruption is about the same over a wide range of high frequency cutoffs. Even two octaves off the bandwidth indicated in the perturbation calculation gives similar end-state results. For $g$ constant, all harmonics are unstable and develop the same microbucket height. A practical benefit of this fact is that tracking with too few particles and too few bins gives approximately the correct emittance growth over somewhat longer time - still only a few beam turns. Therefore, to evaluate the emittance growth to be expected in a particular case, it is not necessary to employ hundreds of millions of macroparticles. A single processor can typically handle $10^{7}$ macroparticles. The number of bins should be fixed so that a reduction in the number of macroparticles by, say, a factor of 2 does not change the result within the desired precision. Something like 1024 bins should be possible.

The self-bunching model stability criterion is consistent with Ng's evaluation of NMI in the MR and also with the FMI examples reported below.

Certainly the consistency of the macroparticle model with the analysis is reassuring, but it is almost surprising in view of the analytical assumption of pure Schottky noise, whereas just $6.4 \times 10^{8}$ macroparticles represent a bunch of $4 \times 10^{10}$ protons in the numerical model. Given that both models have approximations, some further tests are desirable. It is also desirable that the macroparticle model should be validated as much as possible independent of comparisons with the analytical Schottky noise model, because one may want to argue that differing results are evidence of the approximations in the analytical treatment.
The macroparticle model assumes an elliptical phase space distribution, which has a parabolic projection along the beam direction. A parabolic number distribution of $N$ particles with width $2 w$ can be expressed as

$$
n(z)=\frac{3 N}{4 w}\left[1-(z / w)^{2}\right]
$$

The charge distribution of protons is just $\lambda(z)=e n(z)$ and the self-field of the bunch with perfectly conducting wall boundary conditions is

$$
E_{\mathrm{sc}}=-\frac{g_{c} i r c}{4 \pi \varepsilon_{0} \gamma^{2}} \frac{\partial \lambda}{\partial z} .
$$

By restricting the bandwidth of the numerical calculation to $\mathcal{O}(100)$ harmonics, only the field from the bunch envelope is calculated; this can be compared to the result of Eq. (11). The restricted bandwidth calculation of the field of a $0.2 \mathrm{eVs}$ bunch of $2 \times 10^{11}$ protons was repeated with $6.4 \times 10^{8}, 8 \times 10^{7}, 10^{7}$, and $1.25 \times 10^{6}$ macroparticles to assure that the slope of the bunch envelope was precisely determined. The bunch width was taken from the computed charge histogram. The voltage of $\pm 133 \mathrm{kV}$ given by the model agrees to the given number of figures with the voltage calculated by multiplying the field from Eq. (11) by the ring circumference. The code has the capability of calculating the space charge voltage in either frequency or time domain; these largely independent calculations also agree well. Furthermore, the parallelized code used for this modeling agrees with the ESME code[8], which has had several years of shakedown in a wide range of applications.

The results of the restricted bandwidth calculation using 256 bins were the same for macroparticle numbers from $6.4 \times 10^{8}$ to $10^{7}$ but differed by a few percent for $1.25 \times 10^{6}$. Wei has shown for the space charge potential that the number of macroparticles required is proportional to the cube of the number of bins to retain the same statistical noise per bin [9]. From this $n$-cubed binning rule one can infer that $6.4 \times 10^{8}$ macroparticles are also adequate for 2048 bins. The FMI NMI model, however, should have 4096 or more bins to cover the range of Fourier amplitudes predicted to be important by the Schottky noise model. Given the choice of limiting the bandwidth and repeating the history of producing instability at the binning frequency or accepting numerical noise that is possibly too high by $\sqrt{8}$, there is no question that it is more interesting to consider the wider bandwidth. The observation that the $1.25 \times 10^{6}$ macroparticle restricted bandwidth calculation differed from those with higher statistics by about $1 \%$ on the calculated voltage suggests that the final factor of 2 in the binning should not entirely vitiate the results. Another reassuring observation is the agreement of frequency domain and time domain results with 4096 bins per rf period. In addition, results produced at 4096 bins by varying the 
seed for the random number generator agreed within a few percent on emittance growth and strength of the larger Fourier amplitudes.

The checks described are offered as evidence that not only can the macroparticle model be prudently applied for guidance in practical considerations but also it is suitable for examining predictions derived from the analytical Schottky noise model. Both of these points of view will be adopted in specific cases applying to the FMI.

\section{APPLICATION TO THE FERMILAB MAIN INJECTOR}

Generally it is easier to negotiate transition crossing if the longitudinal emittance is small so that single particle nonlinear effects are minimized. A major caveat is that NMI can disrupt the bunch far more than the nonlinearities generally do. Thus, in working out longitudinal impedance budgets one wants to minimize the bunch area approaching transition with the constraint of remaining above the threshold for NMI. Coupled bunch instability stands out as a collective effect that could be exacerbated by small, bright bunches; therefore the capabilities of damper systems are an additional consideration. The NMI threshold, however, is a brick wall lower limit on emittance.
The principal practical outcome of the modeling is summarized in the growth factor vs initial bunch emittance plotted in Fig. 1. The initial parameters are those of a $2 \times 10^{11}$ proton bunch in the FMI. The final emittance is the value just after the nonadiabatic time following transition. The predicted threshold emittance (at fixed intensity) is $0.25 \mathrm{eVs}$. From this plot one can see that NMI is not threatening the present operation at about $6 \times 10^{10}$ protons per bunch at $0.2 \mathrm{eVs}$ pretransition bunch emittance. A better performance in routine operation might be obtained by measures to reduce the bunch area approaching transition to reduce further emittance dilution during transition crossing.

There are two predictions of the analytical Schottky noise model besides the stability threshold which can be rather readily compared to macroparticle model results, viz., the frequency of fastest amplitude growth and the frequency of maximum integrated harmonic growth. In the tabulated results, Table II, the last two columns contain approximations $f_{\text {fast }}$ and $f_{\max }$ of these frequencies estimated visually from plots of the rms amplitudes vs time. These estimates are crude because amplitudes were plotted as rms averages over $3.4 \mathrm{GHz}$ bands, but they suggest that the most active frequencies may be somewhat lower than predicted by the analytical model which gives $f_{\text {fast }}=117 \mathrm{GHz}$ and $f_{\max }=67 \mathrm{GHz}$ in all of these cases. The case of $2 \times 10^{11}$ and $\dot{\gamma}=240$ (line 3 of Table II) was

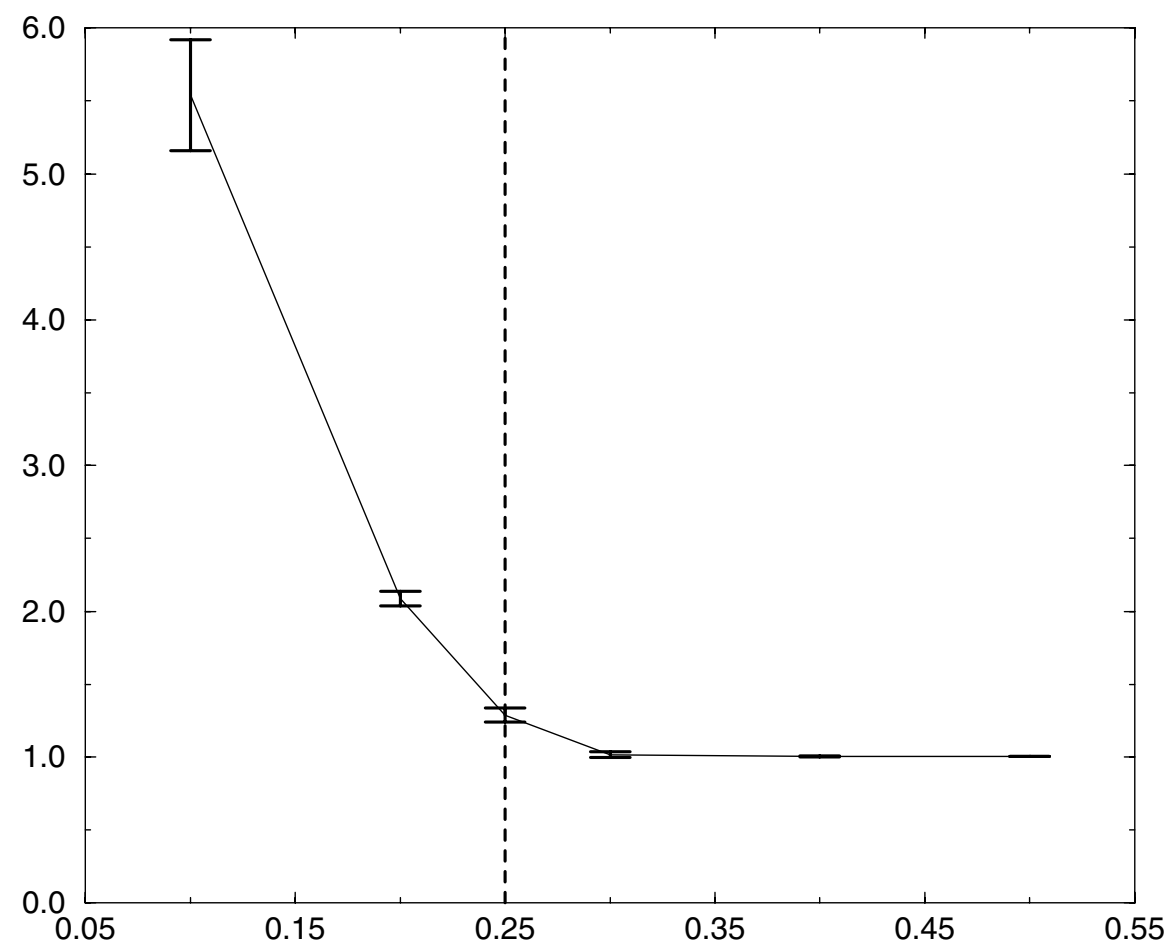

FIG. 1. Emittance growth factor vs initial emittance $(\mathrm{eVs})$ for bunches of $2 \times 10^{11}$ protons crossing transition in the FMI. Each point is determined by a tracking with $6.4 \times 10^{8}$ macroparticles and a run with $10^{8}$. The statistically weighted mean is the central value with error bars given by the difference between the two runs. The vertical dashed line is the NMI threshold evaluated either from Hardt's formulas or the self-bunching criterion. 
TABLE II. Emittance growth for several FMI cases.

\begin{tabular}{cccccc}
\hline \hline $\begin{array}{c}\text { Initial emittance } \\
(\mathrm{eVs})\end{array}$ & $\begin{array}{c}\text { Bunch population } \\
\left(\times 10^{10}\right)\end{array}$ & $\begin{array}{c}\dot{\gamma} \\
\left(\mathrm{s}^{-1}\right)\end{array}$ & $\begin{array}{c}\text { Final emittance } \\
(\mathrm{eVs})\end{array}$ & $\begin{array}{c}f_{\text {fast }} \\
(\mathrm{GHz})\end{array}$ & $\begin{array}{c}f_{\max } \\
(\mathrm{GHz})\end{array}$ \\
\hline 0.2 & 6.0 & 240 & 0.205 & 87 & 56 \\
0.2 & 10.0 & 240 & 0.209 & 97 & 46 \\
0.2 & 20.0 & 240 & 0.411 & 97 & 39 \\
0.2 & 20.0 & 240 & 0.411 & 108 & $62^{\mathrm{a}}$ \\
0.2 & 20.0 & 240 & 0.411 & 107 & $73^{\mathrm{b}}$ \\
0.2 & 10.0 & 120 & 0.221 & 93 & 56 \\
0.2 & 20.0 & 120 & 0.851 & 97 & 46 \\
\hline \hline
\end{tabular}

${ }^{\mathrm{a}}$ See discussion of determination of $f_{\text {fast }}$ and $f_{\max }$ in text.

Equations of motion linearized.

run recording the Fourier spectrum of the beam current every $100 \mu \mathrm{s}$ for the millisecond bracketing transition time. From these plots the harmonics showing the fastest and largest growth can be determined with better precision than for the other cases. Both methods of determining $f_{\text {fast }}$ and $f_{\max }$ were used. The more sensitive method gives the results in line 4 of Table II. To test whether the frequencies are lowered because of nonlinearities in the equations of motion of the macroparticle model, the line 3 case was also run with linear equations of motion except that the rf potential remained sinusoidal. The resulting values obtained with the more sensitive technique are given in line 5 of Table II; they may be directly compared to the values in line 4 for the fully nonlinear calculation. With the better graphical information, the discrepancy between the analytic prediction and the macroparticle model is not gross. Nonetheless, the nonlinear equations of motion apparently do have the hypothesized effect of lowering the dominant frequencies in NMI, especially $f_{\max }$. It is reasonable that $f_{\text {fast }}$ should be less affected because it comes from only the first few time steps of the calculation.

\section{POTENTIAL REFINEMENTS}

There are some tactics for putting the comparison between analysis and macroparticle models on a firmer basis. The most obvious is simply to increase the number of macroparticles. Thirty-two fast PC nodes were used for most of the reported results. A significant improvement in statistics would require something like 10 times this number. A more modest approach is to use a distribution based on a pseudorandom sequence, such as the Sobel sequence. It would become noisier because of the effects of synchrotron oscillation, but by starting the tracking just a few oscillation periods before transition the distribution would still be quieter than a quasirandom distribution of the same number of macroparticles. The strength of the amplitudes can be measured so that the distribution can be fine-tuned for the correct Schottky noise amplitudes at transition. If needed, some pretransition perturbation of the bunch can be avoided by intro- ducing the space charge force gradually. Some such approach or combination of approaches could reduce the numerical noise sufficiently to offer a strong check on the 4096 bin results. It is by no means apparent, however, that such a test is required to support the reported results, nor is it apparent that fine-tuning the distribution is entirely free of bias. Another possibility would be to improve the determination of $f_{\text {fast }}$ and $f_{\max }$ by numerical analysis to permit a more rigorous comparison of the macroparticle results to the analytical values. This latter improvement would have no practical utility.

\section{ACKNOWLEDGMENTS}

This work was supported by the U.S. Department of Energy under Contract No. DE-AC02-76CH03000.

[1] W. Hardt, in Proceedings of the 9th International Conference on High Energy Accelerators (SLAC, Stanford, 1974), pp. 434-438.

[2] K.-Y. Ng, Fermi National Accelerator Laboratory Physics Note No. Fermilab-FN-0713, 2001.

[3] I. Kourbanis and K.-Y. Ng, in Proceedings of the 1993 IEEE Particle Accelerator Conference (IEEE, Washington, DC, 1993), pp. 3630-3632.

[4] G. Jackson et al., in Proceedings of the 1991 IEEE Particle Accelerator Conference (IEEE, San Francisco CA, 1991), pp. 1758-1760.

[5] W.W. Lee and L.C. Teng, in Proceedings of the 8th International Conference on High Energy Accelerators (CERN, Geneva, 1971), pp. 327-330.

[6] J.A. MacLachlan, in Proceedings of the 20th ICFA Advanced Beam Dynamics Workshop, AIP Conf. Proc. No. 642 (AIP, New York, 2002), pp. 68-70.

[7] K. Symon and A. Sessler, in Proceedings of the CERN Symposium on High Energy Accelerators and Pion Physics (CERN, Geneva, Switzerland, 1956), pp. 44-58.

[8] J.A. MacLachlan, in Proceedings of the 17 th International Conference on High Energy Accelerators (Joint Institute for Nuclear Research, Dubna, 1998).

[9] J. Wei, Ph.D. thesis, State University of New York (Stony Brook), 1990. 